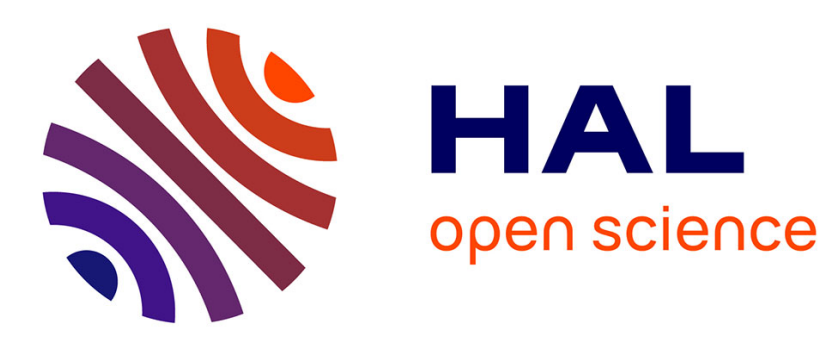

\title{
De la technologie de rupture à la stratégie de rupture: Europlasma, un cas d'entrepreneuriat durable
} Cécile Fonrouge

\section{To cite this version:}

Cécile Fonrouge. De la technologie de rupture à la stratégie de rupture: Europlasma, un cas d'entrepreneuriat durable. Gestion 2000, 2012, 29 (1), pp.39-54. halshs-00687678

\section{HAL Id: halshs-00687678 \\ https://shs.hal.science/halshs-00687678}

Submitted on 13 Apr 2012

HAL is a multi-disciplinary open access archive for the deposit and dissemination of scientific research documents, whether they are published or not. The documents may come from teaching and research institutions in France or abroad, or from public or private research centers.
L'archive ouverte pluridisciplinaire HAL, est destinée au dépôt et à la diffusion de documents scientifiques de niveau recherche, publiés ou non, émanant des établissements d'enseignement et de recherche français ou étrangers, des laboratoires publics ou privés. 


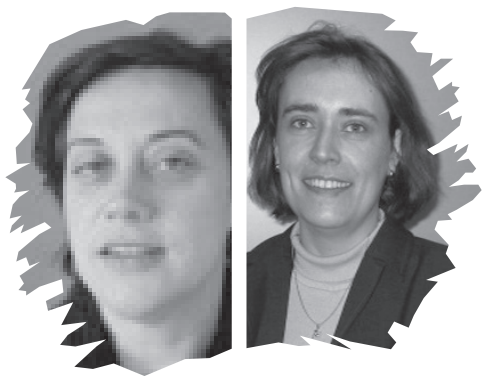

\section{De la technologie de rupture à la stratégie de rupture : Europlasma, un cas d'entrepreneuriat durable}

I es manœuvres stratégiques de certains fabricants d'ordinateurs, de distributeurs Len ligne ou des compagnies aériennes à bas prix ont provoqué un ensemble de réflexions sur les capacités de perturbations que l'on peut avoir en changeant les modèles, les habitudes et les règles du jeu des marchés (Charitou, Markides, 2003; Markides, 2006; Kim, Mauborgne 2009). Si l'on accepte que les changements au sein d'une industrie sont d'autant plus imposants que le secteur était précédemment peu soumis à évolutions alors, il est particulièrement opportun de se pencher sur des secteurs traditionnels pour en observer les bouleversements induits par un petit nombre d'acteurs. Et ne serait-il par encore plus judicieux de se focaliser sur un agent perturbateur qui serait un nouvel acteur de ce marché comme une entreprise nouvellement créée?

C'est le positionnement que nous tenons : étudier une industrie par les yeux d'un nouvel entrant. Autrement dit, comment le secteur traditionnel du traitement des déchets perçoit la « rupture» potentielle apportée par une start-up, Europlasma, et sa technologie révolutionnaire de vitrification des déchets?

La problématique porte sur le passage possible d'une " technologie de rupture » vers une « stratégie » dite de rupture. La première génère des offres non attendues par les clients qui, s'ils sont en position de duopole comme Suez et
Veolia, n'ont pas intérêt à modifier leurs habitudes. La deuxième ou stratégie de rupture remet en cause les modèles et les jeux concurrentiels dans la chaine de valeur et prend en compte la tendance sociétale du développement durable. Notre apport se situe dans cette distinction et dans la délimitation de l'entrepreneuriat durable comme phénomène perturbateur d'un secteur.

Une démarche abductive de recherche fondée sur l'étude d'un cas (Yin, 2009) nous a permis d'approcher en profondeur la compréhension des change- 
ments stratégiques réalisés au sein de I'entreprise Europlasma. Le cas choisi, Europlasma, porte sur le marché des déchets industriels français. II est exemplaire du phénomène à étudier (Hlady-Rispal, 2002) dans la mesure où il s'agit d'une des plus anciennes start-up du secteur qui a suivi, en les anticipant quelquefois, certaines évolutions du secteur comme la valorisation des déchets en énergie. Les données primaires sont constituées d'une observation de 10 mois et de 6 entretiens semi-directifs auprès des principaux responsables de la société (Responsable du développement et responsable du marketing et de la communication). Les données secondaires comprennent la documentation externe disponible et de la documentation interne. La multiplicité des sources de données ont fait l'objet d'un traitement classique d'analyse qualitative (Miles et Huberman, 2003).

Son histoire - voir annexe - montre une technologie de rupture qui cherche à s'imposer comme «design dominant» et a d'abord constitué une impasse stratégique (1). Puis, une volonté stratégique en rupture permet à l'entreprise d'espérer créer un nouvel espace de marché (2) et cela, grâce à son implication dans un processus d'entrepreneuriat durable (3).

Cette démarche séquentielle rend compte de l'aspect processuel des phénomènes de rupture. Elle est préférée à une présentation classique dans la mesure où elle permet de poser une littérature et des conclusions contingentes à la phase traitée.

\section{- Technologie de rupture: impasse d'une recherche de «design dominant»}

Europlasma a été créée en 1992 par Didier Pineau, ancien ingénieur de I'Aérospatiale (EADS). Son idée est d'utiliser la technologie des torches à plasma (cf. encadré 1) dans des applications civiles et notamment dans l'élimination des déchets.

\section{La torche à plasma}

Une torche plasma transforme une énergie électrique en une énergie thermique de haute densité, pouvant atteindre jusqu'à $5000^{\circ} \mathrm{C}$, la température de la surface du soleil. Appliquée au traitement des déchets, une torche à plasma permet l'inertage total de déchets toxiques, leur transformation en en un verre inerte qui peut être valorisé et revendu comme agrégat ou composé de sous-couche routière. Elle peut aussi être utilisée pour l'épuration d'un gaz de synthèse issu de déchets et sa valorisation en production d'électricité. 
La première stratégie d'Europlasma se fonde sur la mise en place d'un standard technologique à partir de cette technologie de rupture.

On parle de technologie de rupture quand la rupture génère des produits dont les attributs sont différents et n'ont pas pu être évalués comme tels par les clients (Walsch, 2004). Pour être rentable, cette technologie, doit être largement diffusée et montrer les caractéristiques d'un "standard » qui s'il s'impose comme mode d'échange inévitable, devient un " design dominant » de marché (1.1). Cette analyse provient des industries de réseaux dont on cherchera à tirer les enseignements pour notre cas (1.2).

\section{Imposer son standard dans les technologies de rupture}

Le standard est assimilé à une norme implicite inter firme qui, hors des frontières de l'entreprise, rythme l'évolution technologique et organisationnelle d'un secteur (Corbel, 2009). En fait, tout se passe comme si une modification dans les critères qui permettent d'assurer la production, l'échange ainsi que la compatibilité des biens et des services jouent le rôle $d$ 'indicateur de changement de régime technologique (Anderson et Tushman, 1990). A la suite des écrits de Kuhn sur la structure des révolutions scienti-

\section{Extrait d'entretien 1}

«Pour les torches à plasma, ... on a commencé par faire l'erreur de s'attaquer aux cendres des incinérateurs qui sont des déchets dangereux car ils contiennent des métaux lourds. Pour l'instant ils sont noyés dans du béton dans des décharges de classe 1. Le coût est deux fois moins élevé que notre solution mais ce facteur n'est pas essentiel. En fait, on ne s'est pas de suite rendu compte que l'on était en plein dans la chaîne de valeur de Suez et Véolia qui produisent les cendres et possèdent les décharges de classe 1. Donc il n'y avait aucune raison, qu'ils nous confient les cendres pour en faire de la sous-couche routière. Tant que la règlementation ne leur défend pas de stocker les cendres malgré leur dangerosité...» (Entretien du 06/11/09 avec Monsieur Lefour, directeur du Développement d'Europlasma)

fiques le standard marque donc une rupture dans le paradigme dominant de l'industrie qui, s'il s'appuie sur une technologie non anticipée dans les besoins des clients, devient une technologie de rupture.

En ce sens, les nouvelles firmes possèdent une supériorité organisationnelle relative par rapport aux entreprises installées. En effet, comme un 
standard modifie la façon de travailler d'une firme en faisant évoluer les structures, programmes, systèmes ou règles internes, il joue sur les routines qui sont encore balbutiantes pour la nouvelle firme. D'où un avantage pour les nouvelles firmes. Mais, pour imposer un nouveau standard, il ne faut pas qu'il y ait contradiction avec des normes existantes de fait ou de droit (Foray, 2002). Europlasma a rencontré des difficultés en France et n'a pas pu s'insérer dans le système en place du traitement des cendres d'incinérateurs $d$ 'ordure ménagère en raison des législations en cours et de la structure de l'industrie.

On voit bien comment, en dépit d'une supériorité technologique, la torche à plasma ne s'impose pas comme procédé $\mathrm{d}^{\prime}$ incinération des métaux lourds car il n'y a pas interdiction de leur stockage.

Cela n'est cependant pas le cas au Japon ou en Corée du Sud où Europlasma s'implante. Au Japon, par exemple, la réglementation est bien plus favorable et il y a beaucoup $d$ 'incinérateurs. Les japonais ont une conscience écologique développée et pas de place pour stocker leur déchet.

Que nous apprennent les industries de réseaux quant aux effets positifs de la mise en place d'un standard?

\section{Les leçons des industries de réseaux}

Le recours au standard est particulièrement adapté aux industries de réseaux et les biens dits "système " comme l'informatique sont les archétypes de ce type de marché. Les réseaux peuvent être physiques ou immatériels : leur point commun est que leur valeur dépend du nombre d'utilisateurs avec un rendement croissant au fil du nombre d'adoptants. Autrement dit, l'action même d'adopter une technologie rend celle-ci plus attractive pour les utilisateurs potentiels, augmentant ainsi ses chances d'être choisie dans le futur (Katz et Shapiro, 1985).

Si la réglementation impose peu quant aux traitements des métaux lourds, ce n'est pas le cas pour l'amiante et Europlasma veut s'imposer comme une solution technique. La firme se voit confortée dans le temps et tire bénéfice de l'exclusion des autres technologies possibles dans un système de rétroaction positive (Foray 2002).

Technologie dominante sur le marché de l'amiante, la solution d'Europlasma constitue une alternative durable qui séduit dans un contexte réglementaire et une tendance sociétale de fond qui influencent la stratégie des clients amenés à dépolluer leur site.

Un autre enseignement porte sur les caractéristiques des industries dans 


\section{Extrait d'entretien 2}

«... on a réussi à se positionner sur une niche autour de l'amiante avec notre site de Morcenx - Inertam. $D^{\prime}$ abord c'est un réel problème de santé publique, (...). Ces déchets amiantés peuvent être stockés en décharge de classe 1 mais surtout 2 quand ils sont classifiés non dangereux. Notre solution est de 3 à 15 fois plus chère mais des propriétaires sont prêts à payer $d$ 'une part parce qu'on leur enlève un problème une fois pour toute et $d^{\prime}$ autre part parce que cela leur fait de la communication positive. Nous ne nous positionnons pas ici contre les intérêts des gros : il se charge du non dangereux avec leur centre d'enfouissement (...) nous on se charge du dangereux.» (Entretien du 06/11/09 avec Monsieur Lefour, directeur du Développement d'Europlasma)

lesquelles les start-up réussissent en imposant leur standard. Selon Anderson et Tushman, «les industries de masse ayant des faibles droits de propriété permettent la domination $d$ 'une architecture unique qui établit une domination d'une certaine classe de produits « (Anderson et Tushman, 1990 p. 619). Une industrie tendant vers le soutenable cherche à se structurer autour d'un « design dominant » car il présente l'avantage de stabiliser les relations entre fournisseurs, clients et partenaires par une protection de type monopolistique. Dans le cas de forts régimes $d$ 'appropriation rendu possible par une politique de propriété industrielle, le marché se fragmente en autant de portions que de propriétaires de brevets - ce qui s'avère contre productifs contrairement à l'organisation autour d'une classe de produits unique.

Or, le secteur du déchet est une industrie de masse dans laquelle des acteurs majeurs sont déjà présents et en situation de quasi duopole. Suez Environnement et Véolia Environne-

\section{Extrait d'entretien 3}

«Pour ce qui concerne les déchets dangereux, les associations contre les décharges et l'incinération des déchets ont aussi joué un rôle dans le resserrage de la concurrence. Les gros rachètent les petits comme par exemple à Bassens où le SIAP où se trouvaient différentes technologies liées au traitement de différents déchets a été racheté par Véolia. Cela permet aux gros acteurs d'intégrer l'ensemble de la filière collecte, tri et élimination des déchets. Les clients préfèrent n'avoir affaire qu'à un seul interlocuteur. « (Entretien du 24/09/09 avec Madame Amiel, Responsable Marketing et Communication d'Europlasma). 
ment compensent leur faible niveau de $R \& D$ - en 2010 , respectivement $0,5 \%$ et $0,4 \%$ du $C A^{1}$ - par une politique d'intégration. Eł dans le cas du marché des déchets dangereux, les besoins poussent les grands acteurs à intégrer l'ensemble de la filière.

Les enseignements des industries de réseaux peinent à s'adapter au marché des déchets en raison du quasi duopole constitué par les deux principaux acteurs qui rigidifient les règles du jeu autour $d^{\prime}$ anciennes technologies. Pour $s^{\prime}$ insérer, les nouvelles firmes exploitent une niche étroite de marché avec l'amiante et passe d'une technologie de rupture à une stratégie de rupture.

\section{Modification de la}

chaîne de valeur et mise en place d'une stratégie de rupture

Europlasma décide de mettre en place le projet $\mathrm{CHO}$-Power soit un nouveau concept de valorisation énergétique des déchets résiduels reposant sur le procédé de la gazéification qui permet de produire de l'électricité à partir de déchets et de biomasse. Celui-ci, constitue une stratégie de rupture (2.1), qui seule, semble permettre à Europlasma $d$ 'assurer son développement sur un marché verrouillé (2.2).

\section{Stratégie de rupture:}

une approche par la valeur

La stratégie de rupture peut être définie comme une stratégie originale qui remet en cause les modèles économiques existants en rebattant les cartes des acteurs et en modifiant les règles du jeu de marché (Stopford et BadenFuller, 1994; Markides, 1997).

Les moyens sont divers : soit on cherche une recombinaison des facteurs clés de succès, pour d'autres cela passera par une réorganisation de la filière ou enfin il est possible de reconfigurer la chaîne de valeur dans son ensemble (Dumoulin et al., 2003, Grandval et Soparnot 2005, Voelpel et al., 2004, Amit et Zott, 2001).

Le résultat est le même : on aboutit à une reformulation du jeu concurrentiel censée perturber les capacités de réactions des concurrents et en particulier des leaders (Hamel, 1997).

Cette stratégie nécessite une remise en cause des fondamentaux : qui sont nos clients? Quels sont nos produits et services? Comment les offrir efficacement? (Markides, 1997). L'innovation stratégique est alors une introduction réussie dans un secteur d'un modèle économique nouveau, né de la modification radicale de la proposition de valeur pour le client

\footnotetext{
1 http://www.suez-environnement.fr et http://
} www.veolia.com (consultés le 08/09/10) 
Figure 1

Modification de

valeur pour le client

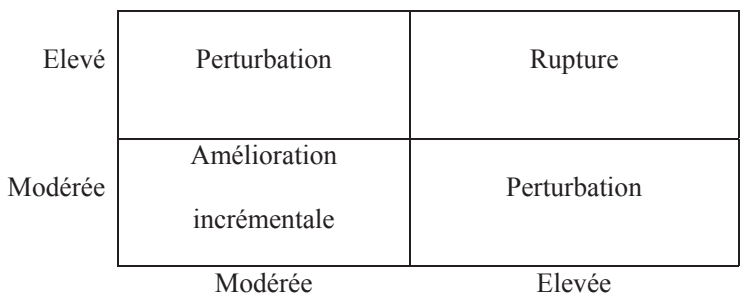

Modification de la chaîne

de valeur

Source : Moingeon et Lehmann-Ortega, 2006.

et/ou de l'architecture de valeur (Lehmann-Ortega et Moingeon, 2010). Découvrir des opportunités d'innovation stratégique revient à remettre en cause l'approche habituelle des clients et de leurs besoins (Markides, 1997 et 2006). II s'agit de détecter la demande latente, celle qui n'est pas exprimée par le client et de décortiquer la courbe de valeur du client en tenant compte de la valorisation par les clients des différents critères d'achat des produits et services existants (Kim et Mauborgne, 1999). La création de valeur peut se faire par la proposition de valeur faite aux clients et par l'architecture de valeur pour la créer (Yip, 2004).

Si l'on suit la piste de la modification de la chaîne de valeur comme levier de rupture, alors on reprendra la matrice (cf. figure 1) qui permet de bien distinguer les améliorations incrémentales de la perturbation et de la rupture en fonction de la modification de valeur pour le client (Moingeon et LehmannOrtega 2006)

Ainsi, pour qu'il y ait rupture, il faut à la fois une modification conséquente de la valeur pour le client et une modification conséquente de la chaîne de valeur. Plus les intensités sont élevées, plus il y a création de changements fondamentaux qui s'éloignent des pratiques existantes et possibilité de création d'un nouveau marché. En intégrant la valorisation des déchets dans sa technologie de destruction, Europlasma traduit cette rupture stratégique. 
De la torche à plasma au projet CHO-Power ou le passage de la technologie de destruction de déchet à sa valorisation en énergie

Avec son projet CHO-Power, Europlasma développe une solution à la fois sur le marché des déchets (alternative à la mise en décharge pour les collecteurs privés) et celui de l'énergie (participation aux objectifs de production d'énergie renouvelable).

La capacité de la centrale électrique CHO-Power est de $12 \mathrm{MWe}$. Elle permet de traiter 55000t/an de biomasse et de déchets locaux. La biomasse concerne les plaquettes forestières du massif landais. Les déchets sont ceux des entreprises tels que les cartons, bois, papiers, tissus, etc., non dangereux. Les collecteurs locaux n'apportent que la fraction résiduelle après leur propre tri et recyclage. Ce flux part aujourd'hui vers des décharges lointaines.

Pour le client cela évite les coûts et les impacts environnementaux du transport et de la mise en décharge. Sa production, vendue à EDF, est de 90000 $\mathrm{MWh} / \mathrm{an}$ ( $=50000$ habitants) peut alimenter le réseau haute tension tout proche.

Europlasma fait émerger une nouvelle architecture de valeur avec deux types de clients dans un même process: les

\section{Extrait d'entretien 4}

«Pour produire de l'électricité à partir des déchets des entreprises, il y a deux manières de le faire. Première manière, les brûler. Mais les combinaisons chimiques sont hasardeuses, cela dégage des polluants cancérigènes (...) Nous, nous proposons la deuxième manière : la transformation en gaz puis en électricité, avec un rendement de $35 \%$. (...) on propose de brûler les déchets que l'on chauffe sans oxygène et donc sans combustion mais qui donne du gaz $\mathrm{CO}+\mathrm{H} 2$, on l'épure avec la torche à plasma, et ce gaz fait tourner des turbines et des moteurs qui donnent de l'électricité. (...) dans un horizon qui a pour objectif de supprimer les décharges, les collecteurs privés ont intérêt à venir chez nous. Les déchets de notre process sont du vitrifiat ou des cendres que l'on peut valoriser en sous-couche routière et moins de $1 \%$ de déchets à mettre en décharge de classe 1 . On est en plein protocole de Tokyo : $20 \%$ de l'énergie doit être renouvelable» (Entretien du 01/10/09 avec Monsieur Lefour, directeur du Développement d'Europlasma.

collecteurs locaux en input du process qui apportent leur déchets à traiter et EDF en output du process qui rachète l'électricité produite. 
CHO-Power est un nouveau concept de valorisation énergétique des déchets résiduels qui accroit les performances énergétiques et environnementales du secteur.

Néanmoins, comme l'ont montré Granval et Soparnot (2005), le développement durable peut constituer en soi une stratégie de rupture $s^{\prime} i l$ conduit à un renouvellement des règles en vigueur et à une création de valeur sur la chaîne de valeur. Les firmes éco-sensibles ont une attitude proactive qui les conduit à construire des ressources et compétences difficiles à imiter donc source d'avantage concurrentiel. Par là même, ils bouleversent le jeu concurrentiel à condition que les parties prenantes accordent une légitimité à ces modifications (Deephouse, 1999).

\section{Entre rupture et insertion dans les habitudes du secteur}

Avec une orientation vers le durable, Europlasma est en rupture avec les habitudes du secteur du traitement des déchets (3.1). Cependant, pour s'intégrer, ce nouvel acteur doit également endosser des rôles et des façons de faire comprises par les autres acteurs (3.2).

\section{L'entrepreneuriat durable: cadre de rupture}

Les caractéristiques de l'entrepreneuriat durable constituent un cadre de rupture exploité par Europlasma.

Dans le courant du « sustainable entrepreneurship » ou entrepreneuriat soutenable ou durable, des entrepreneurs sont réputés poursuivre des opportunités d'affaires liées à l'environnement. On rencontre deux définitions. L'une part des imperfections de marché qui conduisent des entrepreneurs à répondre à ces failles en mettant en place des actions qui réduisent les dégradations faites à l'environnement (Dean et MC Mullen, 2007). De manière proactive et aussi plus large, la deuxième définition s'attache aux déséquilibres environnementaux et à leurs conséquences sociales qui constituent autant de sources d'opportunités d'affaires. Cela permet à certains de croire que la croissance verte sera le vecteur de développement de demain (Cohen et Winn, 2007). Cet attachement aux projections dans l'avenir affilie l'entrepreneuriat soutenable au développement durable qui, depuis le rapport Brundtland du nom de la ministre norvégienne de l'Environnement, décrit: « le développement soutenable comme un développement qui répond aux besoins du présent sans compromettre la capacité des générations futures de répondre aux leurs » (Brunet, 2005 p. 45). 
Ces analyses décrivent un secteur en cours de « verdissement » comme étant celui dans lequel les entreprises prennent en charge un certain nombre de coûts auparavant supportés par la société. Plus exactement, le processus de transformation d'une industrie vers le durable se définit comme l'internalisation des coûts auparavant supportée par l'ensemble de la société civile (Hockerts et Wüstenhagen, 2009). La principale rupture sectorielle se trouve là. Elle se traduit par quatre types de comportement qui permettent de circonscrire l'entrepreneuriat durable : (1) l'état d'esprit d'individus tourné vers l'environnement mais aussi porteurs pour certains de valeurs plus générales; (2) des offres et services évalués en fonction de leur impact sur l'environnement; (3) dans des secteurs orientés vers le durable et enfin avec des stratégies (4) qui se distinguent par leur ancrage institutionnel.

La société Europlasma apparaît impliquée dans un processus d'entrepreneuriat durable car sa situation correspond aux quatre critères définis précédemment:

- Elle se positionne clairement comme un acteur résolument tourné vers l'environnement comme le montre la présentation du groupe sur son site internet

- Les produits et services d'Europlas$\mathrm{ma}$, avec son projet CHO-Power, permettent de transformer une partie des déchets en électricité. On peut donc les évaluer à l'aune des conséquences environnementales qu'ils comportent. Ils répondent à quatre types $d^{\prime}$ imperfection de marché : firmes en place inefficientes, externalités, mécanismes de fixation de prix faussés et asymétrie d'informations (Cohen et Winn, 2007). Rappelons que la majorité des déchets industriels n'est aujourd'hui toujours pas valorisée. Le stockage demeure le mode de traitement le plus couramment utilisé. Le marché du déchet présente des prix fixés : ainsi le coût d'enfouissement de la plupart de ces déchets non dangereux (environ 93\%, Rapport ADEME 2009) en décharge est de $80 €$ la tonne depuis le Grenelle de l'Environnement contre 35 $€$ auparavant.

- La technologie d'Europlasma, en permettant la destruction totale ou la valorisation de déchets jusque là obligés d'être stockés, permet d'internaliser au sein des firmes des coûts supportés précédemment par l'ensemble de la société. En ce sens, elle participe à la transformation de cette industrie vers le durable (Hockerts et Wustenhagen, 2009).

- Son ancrage institutionnel est nécessaire pour développer ses projets comme décrit par l'extrait ci joint. 


\section{Extrait d'entretien $n^{\circ} 5$}

(Ce que font Véolia et Suez)... "c'est du vrai lobbying, nous on ne peut pas influencer la réglementation car il faut des personnes qui $s^{\prime} y$ consacrent à plein temps. Notre influence n'agit qu'au niveau local, nous disposons d'un schéma des personnes à influencer pour assurer la vitesse de la mise en œuvre des projets.» (Entretien du 06/10/09 avec Monsieur Lefour, Responsable du Développement d'Europlasma)

Ce processus d'entrepreneuriat durable permet à Europlasma de s'éloigner des habitudes traditionnelles du secteur pour tenter de trouver un espace plus grand à exploiter. Pourtant, en suivant les courants néo institutionnels être trop différent des habitudes du secteur pose des problèmes qualifiés d'handicap à la nouveauté qu'il faut surmonter.

\section{Etre légitime ou l'adoption de normes partagées}

En adoptant une stratégie de rupture, Europlasma se trouve dans une double crise de légitimité : d'une part une absence de légitimité organisationnelle en raison du jeune âge de la firme et une légitimité institutionnelle en raison de l'absence de consensus sur la stratégie de rupture.

\section{A.L. Stinchcombe donne en 1965 un} point de départ à la notion d'handicap de la nouveauté ou "liability of newness » des entreprises nouvellement créées. Les formalisations historiques d'A.L. Stinchcombe (1972) sont enrichies par celles de leurs successeurs (Singh et al., 1986, Wilkund et al., 2008).

Synthétisant les approches précédentes, on peut déterminer trois dimensions de ce volet externe d'handicap à la nouveauté: le fait d'endosser de nouveaux rôles, d'accéder à des ressources et l'absence, pour la nouvelle firme, d'antériorité dans ses propres relations de marché.

En affirmant sa conception du secteur à travers ses choix stratégiques, Europlasma fait partie des acteurs qui transforment le secteur du traitement des déchets vers le durable. Au regard de sa jeunesse elle doit légitimer ses pratiques.

Les stratégies dans le durable prennent en compte des dimensions institutionnelles fortes ce que Hockerts et Wustenhagen $(2009$, p3) appellent «les stratégies de non marché ». Elles font référence aux stratégies qu'une firme utilise pour influencer l'environnement social et politique. Dans ce contexte, les jeunes firmes font face à un double handicap : celui propres aux marchés émergents et ceux propres à la jeunesse organisationnelle. 
Et, pour qu'une innovation réussisse, elle doit " rencontrer une institution » (Hargandon et Douglas, 2001) et être porté par des acteurs clés qualifiés d'entrepreneurs institutionnels (Maguire et al., 2004).

On décrit trois caractéristiques de ces acteurs:

1. L'occupation de position clés, soit une identité $d^{\prime}$ 'acteurs socialement construite avec une forte légitimité qui permet à l'entrepreneur d'être en lien avec toutes les parties prenantes ;

2. Une capacité qu'ont ces acteurs de théoriser autour du durable. Elle se définit par l'aptitude à relier causalement des pratiques à des résultats particuliers en en montrant les liens logiques ;

3. Un ancrage aux habitudes et aux valeurs du secteur. Il s'agit d'un ensemble de comportements pris par les entrepreneurs pour institutionnaliser ces nouvelles pratiques en les connectant aux routines et aux modes de pensée du champ.

Quels sont les indicateurs de légitimité d'Europlasma? Un premier groupe d'ordre technologique décrit une R\&D en phase avec les recherche sur le plasma et des dépôts de brevets (12 dont 1 CHO-Power) et un second d'ordre relationnel voit émerger l'ensemble des réseaux relationnels en particulier ceux du fondateur et son insertion dans les réseaux locaux (voir l'extrait ci-joint).

\section{Extrait d'entretien 6}

"Aujourd'hui il y a une recherche de productions de plus en plus décentralisées, localisées. Nous, avec notre projet on s'inscrit exactement dans ça : à partir de ressources locales, on propose localement de la production d'électricité. C'est pour ça que l'on a eu un très bon accueil dans les Landes et que ce n'a pas été difficile de convaincre les acteurs locaux.» (Entretien du 19/03/10 avec Madame Amiel, Responsable Marketing et Communication d'Europlasma)

Ainsi, CHO-Power propose une nouvelle pratique connectée aux habitudes du secteur de façon à contrer son double problème de légitimité. D'une part, la rupture apporte des changements fondamentaux qui éloignent des pratiques existantes. De l'autre, pour que l'entreprise soit perçue comme légitime, elle semble devoir s'appuyer sur des acteurs qualifiés d'entrepreneurs institutionnels. 


\section{Conclusion}

Sur un marché traditionnel, quelles sont les capacités de perturbation $d^{\prime}$ une entreprise nouvellement créée à fort contenu technologique? I| s'agit là de la question synthétique à laquelle cette étude de cas répond.

En étudiant Europlasma, nous avons en effet pu observer la volonté de création d'un design dominant à partir d'une technologie de rupture et la manière dont cette intention pouvait constituer, dans notre cas, une impasse.

Pour dépasser ce relatif échec, on propose une analyse en termes de stratégie de rupture. En effet, cette stratégie participe à la création de nouveaux espaces de marché. Elle permet de s'extraire du jeu concurrentiel. Europlasma en a trouvé les conditions de mise en œuvre dans le contexte d'entrepreneuriat durable.

L'entrepreneuriat durable fournit un cadre propice au développement de stratégies de rupture pour les jeunes entreprises au risque de les éloigner des pratiques existantes.

Ainsi, pour qu'elle soit acceptée, cette stratégie de rupture doit être perçue comme légitime à la fois sur le plan organisationnel -en raison du jeune âge de la firme- et sur le plan institutionnel en raison de l'absence de consensus autour d'elle. C'est ici un des premiers enseignements de cette recherche.

Le deuxième met en évidence le fait qu'une PME impliquée dans un processus durable a tout intérêt à s'appuyer sur certains acteurs capables de relier ses pratiques à des résultats attendus et conformes aux habitudes du secteur.

Il reste qu'en entrant par un cas de P.M.E. nous adoptons leurs points de vue de celles-ci dans leur volonté de légitimer le durable. II s'agira de compléter cette recherche par l'étude des grandes entreprises pour lesquelles le « vert » n'est pas seulement un outil de communication.

Ainsi, la piste institutionnelle constitue une direction pour des recherches futures. Et, l'entrepreneur durable à succès devient, certes, celui qui modifie les règles du jeu mais également celui qui se conforme aux institutions du secteur. Nous sommes au cœur d'un paradoxe de la rupture où il s'agit d'être suffisamment différent pour se distinguer sans trop fournir de perturbations pour être tout de même accepté.

\section{- Bibliographie}

ADEME, Rapport 'Les déchets en chiffres en France', 2009 - consultable sur http://www. ademe.fr

AMITT R., ZOTT C., 2001, "Value creation in e-business», Strategic Management Journal, vol. 22, n6/7, pp. 493-520 
ANDERSON, P., TUSHMAN, M., 1990, "Technological discontinuities and dominant designs. A cyclical model of technological change", Administrative Science Quarterly, 35, pp.604-634

BRUNET, S., Le développement durable, PUF,

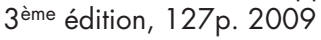

CHARITOU, C.D., MARKIDES C.C., 2003, "Responses to disruptive strategic innovation", MT Sloan management review, winter, pp. 55-63

COHEN, B., WINN M.I., 2007, "Market imperfections, opportunity and sustainable entrepreneurship", Journal of Business Venturing, Vol. 22 Issue 1, pp.29-49

CORBEL P., 2009 Technologie, innovation, stratégie, Gualino, 368p

DEAN T.J., McMULLEN J.S., 2007, "Toward a theory of sustainable entrepreneurship: Reducing environmental degradation through entrepreneurial action", Journal of Business Venturing, Vol. 22 Issue 1, pp.50-76, 2007

DEEPHOUSE D.L., 1999, "To be different or to be the same? It's a question (and theory) of strategic balance", Strategic Management Journal, vol. $20, n^{\circ} 2$, pp. 147-165

DUMOULIN R., METAIS E., PIN R., 2003, "Vers un dépassement de l'antinomie entre rupture ef continuité: le cas Valéo", Xllème Conférence de I'AIMS

FORAY D., 2002, «Innovation et concurrence dans les industries de réseau », Revue française de gestion, 3-4, $n^{\circ} 139$, pp 131-154

GRANDVAL, S., SOPARNOT, R., 2005, «Le développement durable comme stratégie de rupture: une approche par la chaîne de valeur», Management \& Avenir, $n^{\circ} 5$, juillet, pp. 7-26

HAMEL G., 1997, «Réinventer les bases de la concurrence», in GIBSON R., Repenser le futur, Village Mondial, Paris

HARGADON A.B., DOUGLAS Y., 2001, "When innovations meet institutions: Edison and the design of the electric light", Administrative Science Quarterly, 46, pp. 476-501
HLADY-RISPAL, M. (2002), La méthode des cas: application à la recherche en gestion, De Boeck Université, Bruxelles.

HOCKERTS K., WUSTENHAGEN R., 2009, "Greening Goliaths versus emerging Davids Theorizing about the role of incumbents and new entrants in sustainable entrepreneurship", Journal of Business Venturing, Vol. 25 Issue 5, pp. 481-492

KATZ M., SHAPIRO C., 1985, "Network externalities, competition and compatibility", American Economic Review, 75, 3, pp. 424-440

KIM C., MAUBORGNE R., 1999, "Strategy, value innovation, and the knowledge economy", Sloan Management Review, vol. 40, $\mathrm{n}^{\circ} 3$, pp. 41-55

KIM C., MAUBORGNE R., 2009, "How strategy shapes structure", Harvard business review, September, pp. 73-80

LEHMANN-ORTEGA L., MOINGEON B., 2010, "Lever l'incertitude sur les conséquences de l'innovation stratégique", Revue Française de Gestion, $n^{\circ} 203$, pp. $57-70$

MAGUIRE, S., HARDY, C., LAWRENCE, T.B. (2004), "Institutional entrepreneurship in emerging fields ", Academy of Management Journal, Vol. 47, No. 5, p. 657-679

MARKIDES C., 1997, "Strategic innovation", Sloan Management Review, vol. 38, n³, pp. 9-23

MARKIDES C., 2006, "Disruptive innovation: in need of better theory", Journal of product innovation and management, 23, pp. 19-25

MILES, M.B., HUBERMAN, A.M. (2003), Analyse des données qualitatives, De Boeck, Bruxelles.

MOINGEON B., LEHMANN-ORTEGA L., 2006, «Strategic Innovation: How to Grow in Mature Markets», European Business Forum, 24, pp. 50-54

SINGH, J.V., TUCKER, D.J., HOUSE, R.J., 1986, "Organizational legitimacy and the liability of 
newness ", Administrative Science Quarterly, 31, pp. 171-193

STINCHCOMBE, A.L., 1972, Social structure and organizations, in Handbook of organizations, J. March éditor, Rand McNally company, Chicago, 4ème éd. de l'ouvrage de 1965, pp. 142-193

STOPFORD, C., BADEN-FULLER C., 1994, "Creating corporate entrepreneurship", Strategic management journal, Vol. 25, pp. 521-536

VOELPEL S.C., LEIBOLD M., TEKIE E.B., 2004, "The wheel of business model reinvention: how to reshape your business model to leapfrog competitors», Journal of Change Management, vol. $4, n^{\circ} 3$, pp. 259-277
WALSH S.T., 2004, «Roadmapping a disruptive technology: A case study - The emerging Microsystems and top-down nanosystems industry», Technological Forecasting \& Social Change, 71, pp. 161-185

WIKLUND, J., BAKER, T., SHEPHERD, D., 2008, "The age-effect of financial indicators as buffers against the liability of newness", Journal of Business Venturing, in press corrected proof $10,15 p$

YIN R.K. (2009), Case study research: design and methods, Sage, Londres.

YIP G.S., 2004, "Using Strategy to Change Your Business Model", Business Strategy Review, $\mathrm{n}^{\circ} 15$, Issue 2, pp. 17-24 


\section{Annexe 1 : Historique d'Europlasma}

\begin{tabular}{|l|l|}
\hline De 1992 à 2000 & $\begin{array}{l}\text { Mise en œuvre d'une unité pilote équipée de torches à plasma à Cenon (33) } \\
\text { pour démontrer l'efficacité de ses solutions environnementales dans la vitrification } \\
\text { des résidus d'incinération (Il s'agit de la transformation des cendres provenant de } \\
\text { l'incinération des ordures ménagères appelées 'refiom' en verre). }\end{array}$ \\
\hline Depuis 1999 & $\begin{array}{l}\text { Le procédé de la torche à plasma est exploité sur les marchés japonais et coréen } \\
\text { pour la destruction et la valorisation des boues d'épuration urbaines. }\end{array}$ \\
\hline 2001 & $\begin{array}{l}\text { Rachat d'Inertam détenue par EDF. Modernisée, elle est aujourd'hui le seul site } \\
\text { au monde de destruction d'amiante par vitrification. Introduction sur Euronext - } \\
\text { Marché Libre }\end{array}$ \\
\hline Depuis 2004 & $\begin{array}{l}\text { Europlasma travaille sur de nouveaux procédés liés à la conversion de biomasse } \\
\text { (comme le maïs) et de déchets en gaz de synthèse et en électricité. }\end{array}$ \\
\hline 2006 & $\begin{array}{l}\text { Après avoir obtenu la certification ISO 9001, Europlasma prend une participation } \\
\text { dans Europe Environnement unité spécialisée dans le traitement de l'air et des } \\
\text { gaz. }\end{array}$ \\
\hline 2007 & $\begin{array}{l}\text { Europlasma dévoile son projet CHO-Power, usine de production d'électricité à } \\
\text { partir de déchets à Morcenx (40). }\end{array}$ \\
\hline 2009 & Cotation sur Alternext. \\
\hline
\end{tabular}

